\title{
New IR dualities in supersymmetric gauge theory in three dimensions
}

\author{
Micha Berkooz and Anton Kapustin \\ School of Natural Sciences, Institute for Advanced Study \\ Princeton, NJ 08540, USA \\ E-mail: berkooz@sns.ias.edu, kapustinesns.ias.edui
}

\begin{abstract}
We present nontrivial examples of $d=3$ gauge theories with sixteen and eight supercharges which are infrared dual at special points in the moduli space. This duality is distinct from mirror symmetry. To demonstrate duality we construct the gauge theories of interest using D2-branes and orientifolds and then consider their lift to M-theory. We also discuss the strong coupling limit of orientifold two-planes and orbifolds of orientifold six-planes.
\end{abstract}

Keywords: Fiēd Theories in Lower Dimensions, Duality in Gauge Fiè Theories, Mì-Theorȳ' 


\section{Contents}

ii. Introduction

2. $\mathcal{N}=8$ supersymmetric gauge theories in three dimensions

'2.1' IIA brane configurations and the classification of orientifold two-planes

$2 \overline{2}$ Infrared limit and the lift to M-theory

Duality of $\mathcal{N}=8$ gauge theories

3. $\mathcal{N}=4$ supersymmetric gauge theories in three dimensions

'3.1.' IIA brane configurations

M-theory interpretation

is. $\overline{2} \cdot \overline{1} \quad S O(3)$ bundles on $\mathbb{R}^{4} / \mathbb{Z}_{2}$

M-theory on $\mathbb{R}^{4} / \mathbb{Z}_{2} \times \mathbb{R}^{4} / \mathbb{Z}_{2}$

is. $S O(3)$ bundles on $\left(\mathbb{R}^{3} \times S^{1}\right) / \mathbb{Z}_{2}$

M-theory on $\left(\mathbb{R}^{3} \times S^{1}\right) / \mathbb{Z}_{2} \times \mathbb{R}^{4} / \mathbb{Z}_{2}$

Relation to IIA orientifolds

13.3. Duality of $\mathcal{N}=4$ gauge theories

\section{Introduction}

The term "duality" in field theory has several meanings. Electric-magnetic duality is a variable transformation in free abelian gauge theories exchanging the field strength $F$ and its dual $* F$. Strong-weak coupling duality applies to theories which have exactly marginal parameters ("couplings"). It is an equivalence between a strongly coupled theory and another theory which is weakly coupled. For example, an $\mathcal{N}=$ $4 d=4$ gauge theory with gauge group $G$ and complexified gauge coupling $\tau$ is equivalent to an $\mathcal{N}=4$ theory with gauge group $\hat{G}$ (the Langlands dual of $G$ ) and coupling $-1 / \tau$. Yet another type of duality is infrared (IR) duality. Two theories are called IR dual if they flow to the same infrared fixed point. Nontrivial examples in $d=4$ are some $\mathcal{N}=1$ Seiberg dual pairs [ifin. Mirror theories in $d=3[2]$ provide another example of IR duality.

In this letter we present new examples of IR dual theories in $d=3$ with sixteen and eight supercharges. This duality is distinct from mirror symmetry: in all our examples the Coulomb branch moduli of the dual theories are identified, while mirror symmetry exchanges Coulomb and Higgs branches. In the less interesting cases IR duality is visible already at the classical level. For example, at a generic point in the moduli space an $\mathcal{N}=8$ theory with gauge group $G$ flows to a free $\mathcal{N}=8$ theory 
with gauge group $U(1)^{r}$ where $r=\operatorname{rank} G$. Thus any two $\mathcal{N}=8$ theories with gauge groups of equal rank are IR dual at a generic point in the moduli space. The dual pairs discussed in this letter are nontrivial in the sense that their IR equivalence cannot be seen classically.

In section 2 in we show that at a special point in the moduli space an $\mathcal{N}=8$ theory with gauge group $G=S p(2 N)$ is dual to an $\mathcal{N}=8$ theory with $G=O(2 N)$, while at another special point it is dual to an $\mathcal{N}=8$ theory with $G=O(2 N+1)$. The latter duality has been previously derived in [3i]. These dualities hold in the vicinity of orbifold singularities of the moduli space, where classically the full gauge symmetry is restored. Consequently, IR equivalence at these points is a quantum-mechanical phenomenon.

In section 13 , we present an example of IR duality for $\mathcal{N}=4$ theories in $d=3$. We show that at a special point in the moduli space a $U(2 N)$ theory with two fundamentals and two antisymmetric tensors is dual to an $S p(2 N) \times S p(2 N)$ theory with a hypermultiplet in the $(\mathbf{2 N}, \mathbf{2 N})$, a hypermultiplet in the $(\mathbf{2 N}, \mathbf{1})$, and a hypermultiplet in the $(\mathbf{1}, \mathbf{2 N})$. This duality is not visible classically.

To show infrared equivalence we construct the theories of interest using D2branes and orientifolds and then consider the limit of strong IIA coupling. The main idea is that in $d=3$ theories the RG flow is influenced by the VEV of the dual photon. This means that a IIA singularity probed by D2-branes can be resolved into several singularities along the M-theory circle. Theories which look very different in the UV can differ in the IR only by the number and type of singular points. Since the critical IR behavior is determined by the local M-theory geometry, different UV theories may flow to the same IR theory at special points on the moduli space. Similar methods have been used to demonstrate mirror symmetry 胃.

In the case of $\mathcal{N}=8$ theories one needs to understand the strong coupling limit of various $O 2$ planes. The lift of $O 2$ planes to M-theory is studied in section $\mathfrak{2}_{i}^{-}$our discussion there overlaps with that in $[i \overline{3}$. In the case of $\mathcal{N}=4$ theories the relevant

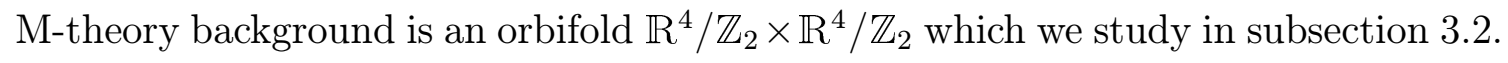

\section{2. $\mathcal{N}=8$ supersymmetric gauge theories in three dimensions}

\subsection{IIA brane configurations and the classification of orientifold two-planes}

Consider $N$ D2-branes parallel to an orientifold 2-plane. The low-energy theory on the D2 worldvolume is an $\mathcal{N}=8 d=3$ gauge theory. The gauge group $G$ depends on the choice of the orientifold projection. If the $\mathrm{RR}$ charge of the $O 2$ plane is positive the gauge group is $S p(2 N)$; if it is negative then $G=O(2 N)$. We will call the former an $\mathrm{O}^{+}$plane, and the latter an $O 2^{-}$plane. Their RR charges are $1 / 8$ and $-1 / 8$, respectively, in the units where the charge of a D2-brane is 1 . In the case of an $O 2^{-}$plane one can also consider adding half of a D2-brane stuck at the fixed point. 
Following [3in] we denote this orientifold plane by $\widetilde{\mathrm{O}}^{+}$; its $\mathrm{RR}$ charge is $3 / 8$. The low-energy theory on D2-branes near an $\widetilde{O} 2^{+}$plane has gauge group $G=O(2 N+1)$.

The above classification of $O 2$ planes was based on the $\mathrm{RR}$ charge. We can also consider a topological classification similar to that in [5] inequivalent ways to quantize a p-brane propagating away from the orientifold plane. Requiring that the p-brane be away from the fixed plane is equivalent to replacing the manifold $\mathbb{R}^{7} / \mathbb{Z}_{2}$ with $\mathbb{R} \times \mathbb{R P}^{6}$ which is homotopic to $\mathbb{R P}^{6}$. Inequivalent ways of assigning phases to disconnected sectors in the p-brane path integral are classified by appropriate (co)homology groups of $\mathbb{R P}^{6}$. The p-branes of interest to us are fundamental strings and D2-branes. Inequivalent ways of choosing phases for a fundamental string path integral are classified by $\operatorname{Hom}\left(H_{2}\left(\mathbb{R P}^{6}, \widetilde{\mathbb{Z}}\right), U(1)\right)=\operatorname{Hom}\left(\mathbb{Z}_{2}, U(1)\right)=$ $\mathbb{Z}_{2} \cdot{ }^{1}$ Equivalently, the possible fluxes of the NS 3-form field strength $H=d B$ are classified by $H^{3}\left(\mathbb{R P}^{6}, \widetilde{\mathbb{Z}}\right)=\mathbb{Z}_{2}$. Since this is pure torsion, the nontrivial cohomology class can be realized by a 2 -form $B$ with vanishing field strength $H$. The only effect of this $B$-field is to multiply certain contributions to the stringy path integral by -1 . More precisely, turning on the $B$-field changes the sign of the contribution of worldsheets wrapping the generator of $H_{2}\left(\mathbb{R P}^{6}, \widetilde{\mathbb{Z}}\right)$ (these have $\mathbb{R P}^{2}$ topology). Recall now that string perturbation theory for D-branes is equivalent to large $N$ expansion in gauge theory [i] and symplectic gauge groups is precisely the sign of the $\mathbb{R P}^{2}$ contributions. Thus we conclude that $\mathrm{O}^{-}$and $\widetilde{\mathrm{O}}^{+}$on one side and $\mathrm{O}^{+}$on the other side are distinguished by discrete torsion in $H^{3}\left(\mathbb{R P}^{6}, \widetilde{\mathbb{Z}}\right)$. We will argue below that it is $O 2^{+}$which has nontrivial torsion.

The other type of p-brane we consider is D2-brane. The choice of phases in the path integral for D2-branes is classified by $\operatorname{Hom}\left(H_{3}\left(\mathbb{R P}^{6}, \mathbb{Z}\right), U(1)\right)=\operatorname{Hom}\left(\mathbb{Z}_{2}, U(1)\right)$ $=\mathbb{Z}_{2}$. By universal coefficient formulas $H^{4}\left(\mathbb{R P}^{6}, \mathbb{Z}\right)=\operatorname{Ext}\left(H_{3}\left(\mathbb{R P}^{6}, \mathbb{Z}\right), \mathbb{Z}\right)=\mathbb{Z}_{2}$, so one can also think of the phase ambiguity as the freedom to choose a cohomology class of the RR 4-form field strength $G^{(4)}$. Since the cohomology is pure torsion, it again can be represented by a $C^{(3)}$ with vanishing field strength. We believe that that $O 2^{-}$and $\widetilde{O 2}{ }^{+}$have different $H^{4}\left(\mathbb{R P}^{6}, \mathbb{Z}\right)$ torsion, but we will not try to prove it here.

\subsection{Infrared limit and the lift to M-theory}

In the extreme infrared the gauge theories in question are described by $N$ M2-branes probing a $d=11$ supergravity background. The moduli space of $N$ D2-branes parallel to an orientifold two-plane is the moduli space of an appropriate $\mathcal{N}=8$

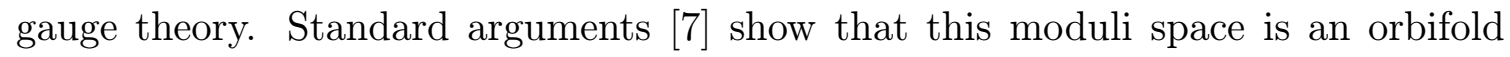
$\operatorname{Sym}\left(\left(\mathbb{R}^{7} \times S^{1}\right) / \mathbb{Z}_{2}\right)^{N}$, hence all $O 2$ planes lift to an M-theory orbifold $\left(\mathbb{R}^{7} \times S^{1}\right) / \mathbb{Z}_{2}$. Let us denote the coordinates on $\mathbb{R}^{7}$ by $x_{1}, \ldots, x_{7}$ and the coordinate on $S^{1}$ by $\sigma$, with

\footnotetext{
${ }^{1}$ Following [iיi] we denote by $\widetilde{\mathbb{Z}}$ a locally constant sheaf of integers whose sections change sign when going around a noncontractible loop in $\mathbb{R P}^{6}$.
} 
the convention that $\sigma$ has period $2 \pi$. There are two points which are left invariant by the orbifold action, $x_{1}=\cdots=x_{7}=\sigma=0$, and $x_{1}=\cdots=x_{7}=0, \sigma=\pi$. We denote them by $p_{+}$and $p_{-}$. We will denote by $m_{+}$(resp. $m_{-}$) the point in the gauge theory moduli space which corresponds to all M2-branes sitting at $p_{+}$(resp. $\left.p_{-}\right)$. Other singular points in the moduli space are obtained if we put $k>0$ of the membranes at $p_{+}$and the rest at $p_{-}$. It is clear that for $k>0$ the resulting SCFT decomposes into a product of two independent SCFT's, one at $p_{+}$and another at $p_{-}$. Both of these SCFT's can be also obtained by starting from a gauge group of lower rank and putting all membranes at the same point. Thus without loss of generality we may concentrate on the situation when all membranes are at the same point.

The neighborhood of either $p_{+}$or $p_{-}$looks like $\mathbb{R}^{8} / \mathbb{Z}_{2}$. There are in fact two different $\mathbb{R}^{8} / \mathbb{Z}_{2}$ orbifolds of M-theory differing by their membrane charge and the cohomology class of the 4 -form field strength $G$. To see this, one can study the possibility of introducing phases in the path integral for membranes. As before, we delete the singular point of $\mathbb{R}^{8} / \mathbb{Z}_{2}$ which makes it homotopic to $\mathbb{R} P^{7}$. The phases are classified by $\operatorname{Hom}\left(H_{3}\left(\mathbb{R P}^{7}, \mathbb{Z}\right), U(1)\right)=\mathbb{Z}_{2}$, which by universal coefficient formulas is related to $H^{4}\left(\mathbb{R} P^{7}, \mathbb{Z}\right)=\mathbb{Z}_{2}$. Nontrivial $H^{4}\left(\mathbb{R P}^{7}, \mathbb{Z}\right)$ torsion means that in the membrane path integral the contribution of membranes wrapped on the cycles homologous to $\mathbb{R P}^{3}$ linearly embedded in $\mathbb{R P}^{7}$ has an extra -1 . We will call the $\mathbb{R}^{8} / \mathbb{Z}_{2}$ orbifolds with and without torsion $\mathrm{B}$ and A-orbifolds, respectively.

It was shown in [3in] that the membrane charge of the A-orbifold (the one without torsion) is $-1 / 16$, while that of the B-orbifold is $3 / 16$. Using this knowledge it is easy to identify the M-theory lifts of all orientifold 2-planes we found above. The $O 2^{-}$plane has RR charge $-1 / 8$ and therefore lifts to an $\left(\mathbb{R}^{7} \times S^{1}\right) / \mathbb{Z}_{2}$ orbifold where both $p_{+}$and $p_{-}$are of type A. The $\widetilde{O 2}{ }^{+}$lifts to an $\left(\mathbb{R}^{7} \times S^{1}\right) / \mathbb{Z}_{2}$ orbifold where both $p_{+}$and $p_{-}$are of type B. Finally, an $O 2^{+}$orientifold has RR charge $1 / 8$ and therefore must lift to an orbifold where one of the points $p_{+}, p_{-}$is of type $\mathrm{A}$ and another is of type B.

Let us now justify the claim that $O 2^{-}$and $\widetilde{O 2}{ }^{+}$planes have trivial $H^{3}\left(\mathbb{R P}^{6}, \widetilde{\mathbb{Z}}\right)$ torsion, while $\mathrm{O}^{+}$has nontrivial torsion. To this end one has to compute the flux of the NS 2-form $B$ through a homology 2-cycle given by $x_{3}=x_{4}=x_{5}=x_{6}=0$. This is the same as computing the flux of the M-theory 3-form $C$ through the M-theory 3 -cycle given by the same formulas. This 3-cycle is homologous to the sum of two small 3-cycles at the points $p_{+}$and $p_{-}$. The fluxes through these 3 -cycles for points of type $\mathrm{A}$ and $\mathrm{B}$ are 0 and $\pi$, respectively. Recalling the M-theory lifts of the $O 2$ planes, we get the desired result.

\subsection{Duality of $\mathcal{N}=8$ gauge theories}

Let us put together the results of the previous two subsections and obtain dualities between $\mathcal{N}=8$ theories with gauge groups $O(2 N), O(2 N+1)$, and $S p(2 N)$. These field theories have special points $m_{+}, m_{-}$on their moduli space where the metric has 
orbifold singularities. At these special points the theories flow to an SCFT described by $N$ membranes near an $\mathbb{R}^{8} / \mathbb{Z}_{2}$ orbifold. The M-theory orbifold can be of type $\mathrm{A}$ or B, and so we can get two distinct SCFT's which will call A and B-models. We showed above that

- The $O(2 N)$ theory flows to the A-model at both $m_{+}$and $m_{-}$.

- The $O(2 N+1)$ theory flows to the B-model at both $m_{+}$and $m_{-}$.

- The $S p(2 N)$ theory flows to the A-model at one point and to the B-model at another point. Which point is which is a matter of convention in field theory. In string theory this information is presumably encoded in the cohomology class of the RR 4-form field strength.

Thus the $S p(2 N)$ theory is dual to both the $O(2 N)$ and the $O(2 N+1)$ theory in the vicinity of certain singular points in the moduli space. The IR equivalence of the $S p(2 N)$ and $O(2 N+1)$ at the origin of the moduli space has been previously shown in [3].

An interesting special case arises if we set $N=1$. The $O(2)$ theory is a $\mathbb{Z}_{2}$ orbifold of the $S O(2)$ theory which is free. Thus for $N=1$ the A-model is a an orbifold of a free SCFT. The $O(3)$ theory, on the other hand, flows to an interacting theory at both $m_{+}$and $m_{-}$[i]i], hence the B-model is interacting. Finally, with a suitable choice of the cohomology class of the RR 4-form field strength the $S p(2)=S U(2)$ theory flows to an interacting fixed point at $m_{+}$and to a free orbifold at $m_{-}$. This agrees with the results of [iiㅔ.

\section{3. $\mathcal{N}=4$ supersymmetric gauge theories in three dimensions}

In this section we will explore IR dualities for $d=3, \mathcal{N}=4$ models. Even though one expects a myriad of $\mathcal{N}=4$ dual pairs, we will restrict ourselves to the simplest singularities in IIA and M-theory. We will be interested in the following two theories:

1. $U(2 N)$ with two hypermultiplet in the antisymmetric tensor representation and two hypermultiplets in the fundamental. For reasons explained below, we shall refer to this theory as the $(A, B)$-model.

2. $S p(2 N) \times S p(2 N)$ with a hypermultiplet in the $(\mathbf{2} \mathbf{N}, \mathbf{2} \mathbf{N})$, a hypermultiplet in the $(\mathbf{2 N}, \mathbf{1})$ and a hypermultiplet in the $(\mathbf{1}, \mathbf{2 N})$. We shall refer to this theory as the $(B, B)$-model.

\subsection{IIA brane configurations}

The IIA backgrounds which the D2-branes probe are given by free worldsheet CFT's. The common ingredients in the CFT's are: 
1. An orientifold 6 -plane which is a point in $x^{7,8,9}$. The corresponding orientifold action, $\Omega^{\prime}$, will be specified more precisely below.

2. A pair (on the base space) of D6-branes branes parallel to the O6-plane.

3. A $\mathbb{Z}_{2}$ orbifold action on the coordinates $x^{3,4,5,6}$ (denoted by $R$ ).

In all cases we will take the orientifold plane to be the $O 6^{-}$plane which has D6-brane charge ${ }^{2}-2$. This implies that we can take [i-i]

$$
\gamma_{\Omega^{\prime}, 6}=1_{4 \times 4}, \quad \gamma_{\Omega^{\prime}, 2}=\left(\begin{array}{cc}
0 & 1_{2 N \times 2 N} \\
-1_{2 N \times 2 N} & 0
\end{array}\right)
$$

where there are $N$ physical D2-branes.

We now have the freedom to choose $[\overline{9}]]$ the sign $\eta$ in the equation

$$
\gamma_{\Omega^{\prime} R, 6}=\eta \gamma_{\Omega^{\prime} R, 6}^{T}, \quad \gamma_{\Omega^{\prime} R, 2}=-\eta \gamma_{\Omega^{\prime} R, 2}^{T} .
$$

This choice is correlated with the action of $\Omega^{\prime}$ on the twisted sector states of $R$. The twisted sector states are a RR vector field $A_{0,1,2}^{(1)}, 3$ NSNS scalars $\phi_{i}$, a RR vector $A_{7,8,9}^{(2)}$ and an NSNS scalar $\phi_{0}$. For $\eta=-11+\Omega^{\prime}$ projects (at the intersection of the orientifold and orbifold) onto $A^{(1)}$ and $\phi_{i}$, and for $\eta=1$ it projects onto $A^{(2)}$ and $\phi_{0}$.

The orbifold with $\eta=-1$ is T-dual to the Gimon-Polchinski model. A convenient choice for the projection matrices is:

$$
\gamma_{R, 6}=\left(\begin{array}{c}
1_{2 \times 2} \\
-1_{2 \times 2}
\end{array}\right), \quad \gamma_{R, 2}=\left(\begin{array}{c}
\sigma_{1} \\
-\sigma_{1}
\end{array}\right) .
$$

This gives rise to the $(A, B)$ model on the D2 worldvolume.

For $\eta=1$, a convenient choice of matrices is:

$$
\gamma_{R, 6}=\left(\begin{array}{cc}
1_{2 \times 2} & \\
& -1_{2 \times 2}
\end{array}\right), \quad \gamma_{R, 2}=\left(\begin{array}{c}
i \sigma_{2} \\
-i \sigma_{2}
\end{array}\right) .
$$

This gives rise to the $(B, B)$ model on the D2 worldvolume. $\gamma_{R, 2}$ has to be traceless in order to cancel an unphysical twisted tadpole.

Before the $R$ projection, the choice ( $(\overline{3} . \overline{1})$ determines the Lie algebra of the gauge symmetry on the D6-branes to be $S O(4)$. Choosing $\gamma_{R, 6}$ is equivalent to specifying a monodromy of a flat connection on $\mathbb{R}^{4} / \mathbb{Z}_{2}$ with the origin removed. It is easy to see that in the $(A, B)$ model the gauge bundle does not admit a vector structure [1] (see also [1] [1] $\left.]_{1}^{1}\right]$, while in the $(B, B)$ model it admits a vector structure but no spinor structure. This suggests that the bundle is actually an $S O(3) \times S O(3)$ bundle.

\footnotetext{
${ }^{2}$ Charges will always be counted on the base space.
} 


\subsection{M-theory interpretation}

Both the $(A, B)$ and $(B, B)$ models lift to $\mathrm{N}$ M2-branes probing M-theory on $\mathbb{R}^{4} / \mathbb{Z}_{2} \times$ $\left(\mathbb{R}^{3} \times S^{1}\right) / \mathbb{Z}_{2}$. This background has two points that are fixed by the entire orbifold group: one at $x^{3 \ldots 9}=0, \sigma=0$ and another at $x^{3 \ldots 9}=0, \sigma=\pi$. As before, we will denote these points by $p_{+}$and $p_{-}$. The overall picture is that of two parallel 7-planes of $A_{1}$ singularities which are intersected by an orthogonal 7-plane of $A_{1}$ singularities. The local behavior near each intersection is therefore of the form $\mathbb{R}^{4} / \mathbb{Z}_{2} \times \mathbb{R}^{4} / \mathbb{Z}_{2}$. Since we have three $A_{1}$ planes, one would expect to have three $s u(2)$ gauge multiplets. A pair of $s u(2)$ 's that lives on the parallel $A_{1}$ 's corresponds to the $s o(4)$ multiplet on the D6-branes. The third su(2), however, must be broken by a Wilson line if we are to obtain a perturbative type IIA model. This will explained in greater detail below.

The two parallel $A_{1}$ singularities wrap $\mathbb{R}^{4} / \mathbb{Z}_{2}$. Supersymmetric vacua correspond to self-dual $S O(3) \times S O(3)$ connections on $\mathbb{R}^{4} / \mathbb{Z}_{2}$. Instantons on this space were discussed in 101, and we remind the reader the salient features of that discussion below. The remaining $A_{1}$ wraps $\left(\mathbb{R}^{3} \times S^{1}\right) / \mathbb{Z}_{2}$ necessitating the analysis of $S O(3)$ bundles on this space. The situation is further complicated by the fact that the monodromies on the the two spaces are correlated, a fact which we explain below.

The net result will be that there are two types of $\mathbb{R}^{4} / \mathbb{Z}_{2} \times \mathbb{R}^{4} / \mathbb{Z}_{2}$ singularities, which we will call $A$ and $B$-singularities. An astute reader no doubt anticipates that the $(A, B)$ model contains one of each singularities, while the $(B, B)$ model contains two $B$-singularities. The astute reader is correct.

\subsection{1 $S O(3)$ bundles on $\mathbb{R}^{4} / \mathbb{Z}_{2}$}

For our purposes it is sufficient to consider point-like instantons stuck at the fixed point of the orbifold. Arbitrary instantons can be obtained by combining such an object with ordinary instantons which are free to roam on $\mathbb{R}^{4} / \mathbb{Z}_{2}$. From the string theory point of view, ordinary instantons are simply D2-branes stuck to D6-branes; we can always shrink them to zero size and move off the $A_{1}$ singularity.

The stuck instanton is flat everywhere except the origin. Let us denote $\mathbb{R}^{4} / \mathbb{Z}_{2}$ with the origin removed by $\widetilde{\mathbb{R}^{4} / \mathbb{Z}_{2}}$. The fundamental group of $\widetilde{\mathbb{R}^{4} / \mathbb{Z}_{2}}$ is $\mathbb{Z}_{2}$; flat connections are classified by the conjugacy class of monodromy around the generator of this $\mathbb{Z}_{2}$. Up to conjugacy, there are two possible monodromies, the trivial one and the one given by

$$
U=\left(\begin{array}{lll}
-1 & & \\
& -1 & \\
& & 1
\end{array}\right) .
$$

If the monodromy is conjugate to $U$, the parallel transport for spinors of $S O(3)$ cannot be defined: this is an $S O(3)$ connection without spinor structure.

An alternative way of thinking about the stuck instanton is to blow-up the singularity slightly, making $\mathbb{R}^{4} / \mathbb{Z}_{2}$ into a Eguchi-Hanson space $M_{E H}$. Then the 
instanton becomes a bona fide self-dual connection on $M_{E H}$. In the case of trivial monodromy it is a trivial connection. In the case of nontrivial monodromy it is a connection with a nontrivial second Stiefel-Whitney class. Such a connection can be constructed by embedding a particular $U(1)$ instanton on $M_{E H}$ into $S O(3)$ [i] 10 . Its topological charge is $1 / 8$ of the charge of a "free" $S O(3)$ instanton.

\subsubsection{M-theory on $\mathbb{R}^{4} / \mathbb{Z}_{2} \times \mathbb{R}^{4} / \mathbb{Z}_{2}$}

As explained above, the local geometry near either $p_{+}$or $p_{-}$is that of two orthogonal planes of $A_{1}$ singularities, i.e. $\mathbb{R}^{4} / \mathbb{Z}_{2} \times \mathbb{R}^{4} / \mathbb{Z}_{2}$. We will label the coordinates of the first $\mathbb{R}^{4} / \mathbb{Z}_{2}$ by $x^{\alpha, \beta, \ldots}$ and of the second one by $y^{\mu, \nu, \ldots}$. It follows that we have two $S O(3)$ bundles, one living at $x=0$ and another one at $y=0$. Naively the bundles appear independent, but we as we will now see this is not the case.

From the previous subsection we know that after a slight blow-up a nontrivial $S O(3)$ bundle is characterized by a self-dual field strength $F$ in some $U(1)$ subgroup of $S O(3)$. This $U(1)$ subgroup can be chosen so that the $U(1)$ gauge field $A(y)$ corresponds to a $C$-field of the form $C(x, y) \sim A(y) \wedge w(x)$, where $w(x)$ is the selfdual 2-form on $M_{E H}$ representing an integer cohomology class. Thus a non-trivial $F$ corresponds to turning on a field strength $G=d C$ of the form

$$
\frac{G}{2 \pi}=w(x) \wedge w(y)
$$

i.e. to a flux of $G$ through the 4-cycle dual to $w(x) \wedge w(y)$. The constant of proportionality in (…… cohomology class.

Note that this condition is symmetric under the interchange of the two $\mathbb{R}^{4} / \mathbb{Z}_{2}$ 's. This implies that if we have a nontrivial $S O(3)$ monodromy on one $\mathbb{R}^{4} / \mathbb{Z}_{2}$, then there is also a similar nontrivial monodromy on the other one. Therefore there are only two types of $\mathbb{R}^{4} / \mathbb{Z}_{2} \times \mathbb{R}^{4} / \mathbb{Z}_{2}$ orbifolds in M-theory. We will call the one with trivial monodromies an $A$-singularity, and the one with both monodromies equal to $U$ a $B$-singularity.

For future use, let us compute the membrane charge of both types of $\mathbb{R}^{4} / \mathbb{Z}_{2} \times$ $\mathbb{R}^{4} / \mathbb{Z}_{2}$ orbifolds. For the $A$-singularity the charge comes from a $C \wedge X_{8}(R)$ interaction in $d=11$ supergravity, while for the $B$-singularity there is also a contribution from the Chern-Simons term $C \wedge G \wedge G$. The gravitational contribution to the charge is simply $-\chi / 24$, where $\chi$ is the integral of the Euler density. To compute $\chi$ we consider a compactified orbifold $T^{4} / \mathbb{Z}_{2} \times T^{4} / \mathbb{Z}_{2}$ whose Euler number is $2^{8}$ times the Euler number of $\mathbb{R}^{4} / \mathbb{Z}_{2} \times \mathbb{R}^{4} / \mathbb{Z}_{2}$. The compactified orbifold can be blown up to $K 3 \times K 3$, so its Euler number is $24^{2}$. It follows that the integral of the Euler density for $\mathbb{R}^{4} / \mathbb{Z}_{2} \times \mathbb{R}^{4} / \mathbb{Z}_{2}$ is $9 / 4$, and the gravitational contribution to the membrane charge is $-3 / 32$. When the $G$ field of the form $\left({ }_{3} . \overline{6}_{1}^{\prime}\right)$ is switched on, there is an additional 
contribution to the charge

$$
\frac{1}{2} \int \frac{G}{2 \pi} \wedge \frac{G}{2 \pi}
$$

Since

$$
\int_{M_{E H}} w(x) \wedge w(x)=-\frac{1}{2},
$$

this contribution is equal to $1 / 8$. Thus the membrane charges of $A$ and $B$-singularities are $-3 / 32$ and $1 / 32$, respectively.

\subsection{3 $S O(3)$ bundles on $\left(\mathbb{R}^{3} \times S^{1}\right) / \mathbb{Z}_{2}$}

Our orbifold $\left(\mathbb{R}^{3} \times S^{1}\right) / \mathbb{Z}_{2} \times \mathbb{R}^{4} / \mathbb{Z}_{2}$ contains a submanifold of $A_{1}$ singularities which wraps $\left(\mathbb{R}^{3} \times S^{1}\right) / \mathbb{Z}_{2}$. Thus we need to understand self-dual $S O(3)$ bundles on this space. When the orbifold singularities in this space are removed, the question reduces to the analysis of flat $S O(3)$ bundles. These are classified by homomorphisms from the fundamental group to $S O(3)$ modulo conjugation.

The computation of the fundamental group is illustrated in figure $1-$ In this figure $z=x^{1}+i x^{2}$ and $w=e^{x^{3}+i x^{4}}$, with $x^{1 \ldots 3}$ being the affine coordinates on $\mathbb{R}^{3}$, and $x^{4}$ being the angular coordinate on $S^{1}$ with period $2 \pi$. The origin of the $w$-plane is excised for every value of $z$. The $\mathbb{Z}_{2}$ projection acts as $z \rightarrow-z, w \rightarrow 1 / w$. The points $(w, z)=(1,0)$ and $(-1,0)$, denoted by " $\mathrm{x}$ ", are the fixed points of this action and are removed as well. It is clear from the figure that the generators $c_{1}, c_{2}, a$ of the fundamental group satisfy

$$
c_{1} c_{2}=a, c_{1}^{2}=c_{2}^{2}=1, c_{1} a c_{1}=a^{-1} .
$$

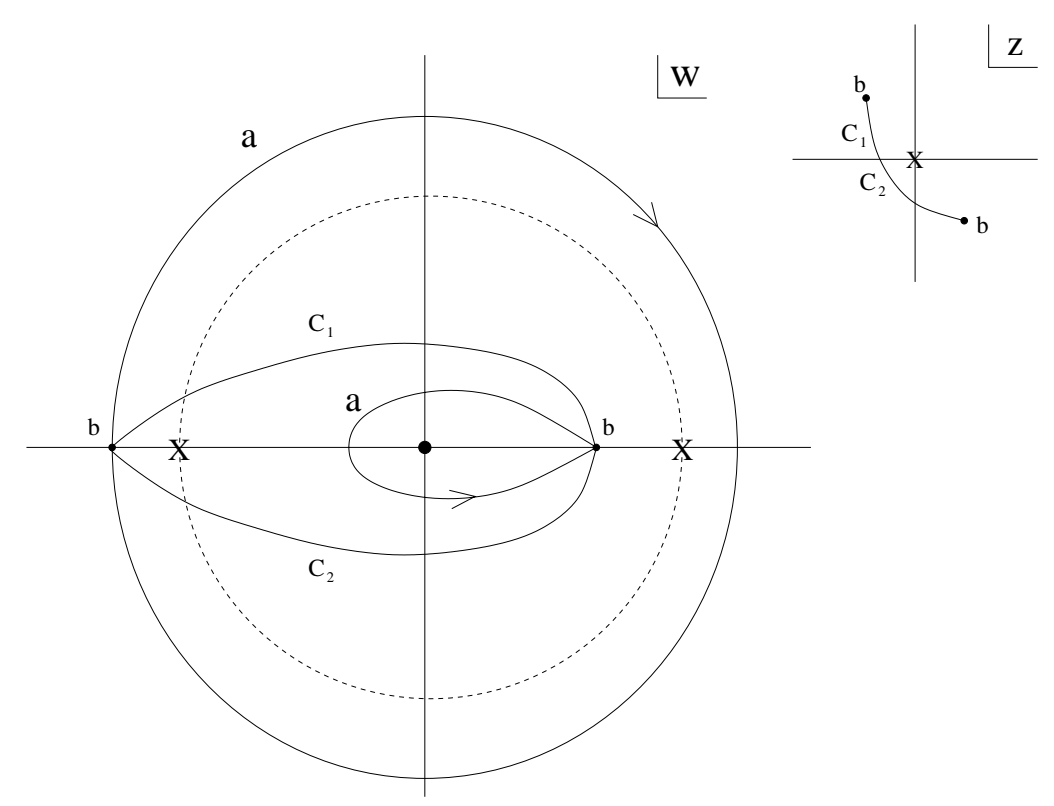

Figure 1: Calculation of the fundamental group of $\left(\mathbb{R}^{3} \times S^{1}\right) / \mathbb{Z}_{2}$ with two singular points deleted. 
$c_{2}$ can be eliminated using these relations. However, it is useful to keep it since Wilson lines along $c_{1}$ and $c_{2}$ measure the monodromy around the two (symmetric) fixed points. The homomorphisms from this fundamental group to $S O(3)$ fall into three classes:

1. $W\left(c_{1}\right)=W\left(c_{2}\right)=W\left(c_{3}\right)=1_{3 \times 3}$,

2. $W\left(c_{1}\right)=1_{3 \times 3}, W\left(c_{2}\right)=U, W(a)=U$,

3. $W\left(c_{1}\right)=U, W\left(c_{2}\right)=\mathcal{R}(\phi) U \mathcal{R}(\phi)^{-1}, W(a)=U \mathcal{R}(\phi) U \mathcal{R}(\phi)^{-1}$,

where

$$
\mathcal{R}(\phi)=\left(\begin{array}{ccc}
1 & & \\
& \cos \frac{\phi}{2} & \sin \frac{\phi}{2} \\
& -\sin \frac{\phi}{2} & \cos \frac{\phi}{2}
\end{array}\right) .
$$

Note that the third class is a one-parameter family indexed by $\phi \in[0, \pi]$.

3.2.4 M-theory on $\left(\mathbb{R}^{3} \times S^{1}\right) / \mathbb{Z}_{2} \times \mathbb{R}^{4} / \mathbb{Z}_{2}$

Putting all of this together, we come to the following classification of $\left(\mathbb{R}^{3} \times S^{1}\right) / \mathbb{Z}_{2} \times$ $\mathbb{R}^{4} / \mathbb{Z}_{2}$ orbifolds in M-theory. There are three types of such orbifolds corresponding to three types of flat $S O(3)$ bundle on $\left(\mathbb{R}^{3} \times S^{1}\right) / \mathbb{Z}_{2}$. Picking a particular type of the bundle also fixes the monodromy of the flat $S O(3) \times S O(3)$ bundle which lives on $\mathbb{R}^{4} / \mathbb{Z}_{2}$. For the three choices described in the previous subsection the $S O(3) \times S O(3)$ monodromies are

$$
1:\left(1_{3 \times 3}, 1_{3 \times 3}\right), \quad 2:\left(1_{3 \times 3}, U\right), \quad 3:(U, U) .
$$

In the first case there are two $A$-singularities, in the second case there is one $A$ and one $B$-singularity, and in the third case there are two $B$-singularities. In the first case $S O(3) \times S O(3)$ gauge group is not broken by the monodromy, in the second case it is broken down to $U(1) \times S O(3)$, and in the third case it is broken down to $U(1) \times U(1)$. It is also important to know how the monodromies on $\left(\mathbb{R}^{3} \times S^{1}\right) / \mathbb{Z}_{2}$ break the $S O(3)$ which lives there. The unbroken group consists of all elements of $S O(3)$ which commute with all the monodromies. In the $(A, A)$ case the $S O(3)$ is unbroken, and in the $(A, B)$ it is broken down to $U(1)$. In the $(B, B)$ case the $S O(3)$ is completely broken for generic values of the parameter $\phi$, while for $\phi=0, \pi$ there is a residual $U(1)$.

\subsubsection{Relation to IIA orientifolds}

In the weak coupling limit the two parallel planes of $A_{1}$ singularities in M-theory become an $O 6^{-}$plane and a pair of D6-branes. The $S O(3) \times S O(3)$ gauge bundle becomes the gauge bundle on D6-branes. As discussed in subsection is. for the $(A, B)$ and $(B, B)$ orientifolds the gauge bosons on D6-branes are in the adjoint of 
$u(2)$ and $u(1) \times u(1)$, respectively. Hence the $(A, B)$ orientifold must be the weak coupling limit of the $(A, B)$ singularity in M-theory, while the $(B, B)$ orientifold comes from the $(B, B)$ singularity.

Note that there is no IIA orientifold corresponding to the $(A, A)$ singularity. The reason is that in the $(A, A)$ case there is an unbroken nonabelian gauge group living at the origin of the $\mathbb{R}^{4} / \mathbb{Z}_{2}$ orbifold. This gauge group is nonperturbative from the IIA point of view, hence the corrresponding type IIA background cannot be described by a free worldsheet CFT. As explained in [12i], perturbative orbifolds avoid gauge symmetry enhancement by assigning a nonzero expectation value to a certain scalar in the twisted NSNS sector. In the M-theory language, the breaking of the symmetry is due to an $S O(3)$ Wilson line along the M-theory circle; the above-mentioned scalar parametrizes its eigenvalues. In the $(A, A)$ case the Wilson line $W(a)$ is frozen at 1 , so no description based on a free orbifold is possible. In the $(A, B)$ case the Wilson line is frozen at a nontrivial value which breaks $S O(3)$ down to $U(1)$. In the $(B, B)$ case the Wilson line is parametrized by a real variable $\phi \in[0, \pi]$ which is identified with the twisted NSNS scalar $\phi_{0}$ in IIA (see subsection ' $3 . \overline{1}^{\prime}$ ). The perturbative IIA construction picks a particular value for the $\mathrm{VEV}$ of $\phi_{0}$; one can argue that this value is $\pi / 2$.

As a check of this identification of M-theory orbifolds and IIA orientifolds, let us compare their membrane charges. According to subsection 3.2 .24 the M2-brane charges of $(A, B)$ and $(B, B)$ orbifolds are $-1 / 16$ and $1 / 16$, respectively. The membrane charge of perturbative IIA orientifolds can be determined by studying tadpoles. Alternatively, we can make use of the fact that the $(A, B)$ model is T-dual to the Gimon-Polchinski model [8], while the $(B, B)$ model is T-dual to the orientifold constructed in [ind. The charge of the former is $-1 / 2$, while that of the latter is $1 / 2$. T-duality along three directions parallel to the orientifold planes reduces the charges by a factor of 8 , giving $-1 / 16$ and $1 / 16$. Thus we find complete agreement between the perturbative IIA computation and the supergravity computation in $d=11$.

\subsection{Duality of $\mathcal{N}=4$ gauge theories}

From the M-theory description it follows that the moduli space of both $(A, B)$ and $(B, B)$ gauge theories is an orbifold $\operatorname{Sym}\left(\left(\mathbb{R}^{3} \times S^{1}\right) / \mathbb{Z}_{2} \times \mathbb{R}^{4} / \mathbb{Z}_{2}\right)^{N}$. This orbifold has $2^{N}$ fixed points. We denote by $m_{+}, m_{-}$the two points on the moduli space corresponding to all M2-branes at $p_{+}$or $p_{-}$. Just as in the $\mathcal{N}=8$ case, it is sufficient to examine the theory at $m_{+}, m_{-}$.

The discussion in subsection IR fixed point both at $m_{+}$and $m_{-}$. This fixed point has a conserved $u(1)$ current. The $(A, B)$ model flows to inequivalent fixed points at $m_{+}$and $m_{-}$. One of them has a conserved $u(1)$, while the other one has an $s u(2)$ current algebra. The fixed point with the $u(1)$ current is the same as the fixed point to which the $(B, B)$ model flows. 
Classically, the full gauge symmetry is restored when the D2-branes sit on top of the orientifold plane, irrespective of the VEVs of the dual photons. Therefore the IR equivalence of $(A, B)$ and $(B, B)$ models in the vicinity of orbifold singularities is a quantum-mechanical phenomenon.

There exists yet another $\mathcal{N}=4$ gauge theory which flows to the fixed point with a conserved $u(1)$ and therefore is IR dual to both $(A, B)$ and $(B, B)$ models. It is a $U(N) \times U(N)$ theory with a four hypermultiplets in the representations $(\mathbf{N}, \overline{\mathbf{N}}),(\overline{\mathbf{N}}, \mathbf{N}),(\mathbf{1}, \mathbf{N})$, and $(\mathbf{N}, \mathbf{1})$. This theory arises on D2-branes probing two D6-branes wrapped on $\mathbb{R}^{4} / \mathbb{Z}_{2}$. If $\mathbb{Z}_{2}$ acts on D2 Chan-Paton labels by

$$
\gamma_{R, 2}=\left(\begin{array}{cc}
1_{N \times N} & \\
& -1_{N \times N}
\end{array}\right),
$$

cancellation of unphysical tadpoles requires

$$
\gamma_{R, 6}=\left(\begin{array}{cc}
1 & \\
& -1
\end{array}\right)
$$

It is easy to see that the theory on the probes has the gauge group and matter content described above. This IIA background lifts to $M_{T N 2} \times \mathbb{R}^{4} / \mathbb{Z}_{2}$ in M-theory, where $M_{T N 2}$ is a two-center Taub-NUT space with coincident centers. $M_{T N 2}$ is topologically equivalent to $\mathbb{R}^{4} / \mathbb{Z}_{2}$, therefore we are dealing with an $\mathbb{R}^{4} / \mathbb{Z}_{2} \times \mathbb{R}^{4} / \mathbb{Z}_{2}$

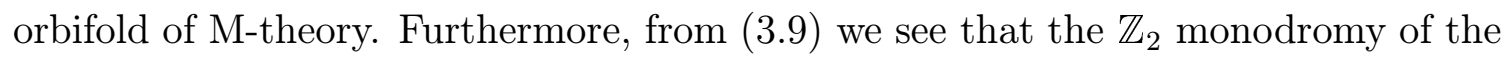
D6 bundle breaks the $\mathrm{D} 6$ gauge group from $U(2)$ down to $U(1) \times U(1)$. From the M-theory point of view, the diagonal $U(1)$ comes from the untwisted sector, while the difference of the two $U(1)$ 's comes from the twisted sector. Recall now that for $A$ and $B$ singularities the twisted sector gauge group is $S O(3)$ and $U(1)$, respectively. We conclude that the IIA orbifold with Chan-Paton matrices as in ( (13. $\left.\bar{z}_{-}^{\prime}\right)$, ( ( to a singularity of type $B$. It follows that at the origin of the moduli space the probe theory is IR dual to both $(B, B)$ and $(A, B)$ models.

\section{Acknowledgments}

We would like to thank J. Park, N. Seiberg, S. Sethi, and E. Witten for helpful discussions. We are especially grateful to K. Intriligator for patient explanations of his work. The work of M.B. is supported by NSF grant PHY-9513835; that of A.K. by DOE grant DE-FG02-90ER40542.

\section{References}

[1] N. Seiberg, Electric-Magnetic Duality in Supersymmetric Nonabelian Gauge Theories, iNucl. Phys. B $435(1995)$ 129i [nep-th/9411149'].

[2] N. Seiberg and K. Intriligator, Mirror Symmetry in Three-Dimensional Gauge Theo-

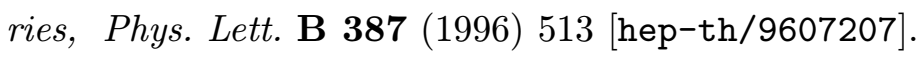


[3] S. Sethi, A Relation Between $N=8$ Gauge Theories in Three Dimensions,

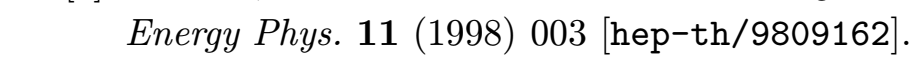

[4] M. Porrati and A. Zaffaroni, $M$ Theory Origin of Mirror Symmetry in ThreeDimensional Gauge Theories, Nucl. Phys. B 490 (1997) 107] [hep-th/9611201'].

[5] E. Witten, Baryons and Branes in Anti de Sitter Space, iJ. High Energy Phys. 07.

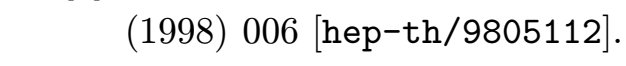

[6] M. Bershadsky, Z. Kakushadze and C. Vafa, String Expansion as Large $N$ Expansion

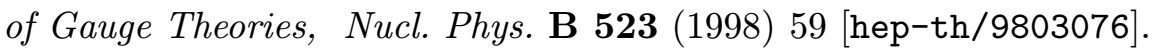

[7] N. Seiberg, Notes on Theories with Sixteen Supercharges, iNucl.

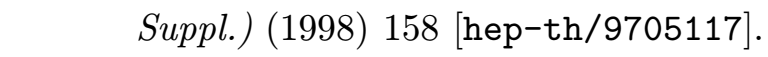

[8] E.G. Gimon and J. Polchinski, Consistency Conditions for Orientifolds and DManifolds, 'Phys. Rev. D 54 (1996) 166\% [hep-th/9601038'.

[9] J. Polchinski, Tensors from K3 Orientifolds, 'P $\bar{h} y s$. [hep-th/9606165i.

[10] M. Berkooz, R.G. Leigh, J. Polchinski, J.H. Schwarz and E. Witten, Anomalies, Dualities, and Topology of $D=6 \mathrm{~N}=1$ Superstring Vacua, hep-th/9605184.

[11] K. Intriligator, RG Fixed Points in Six Dimensions via Branes at Orbifold Singularities, iNucl. Phys. B 496 (1997) 177; [hep-th/9702038'].

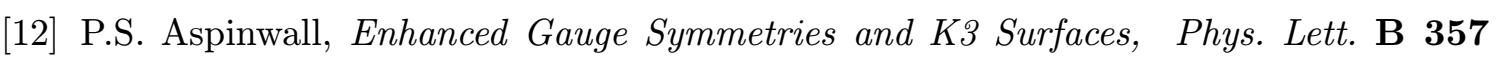

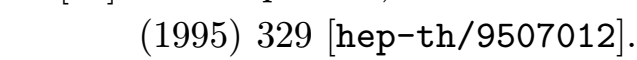

